\title{
Plantas Daninhas na Pós-Colheita de Milho nas Várzeas do Rio São Francisco, em Minas Gerais ${ }^{1}$
}

\author{
Weed Survey on Corn Post-Harvest Under Meadow Holm Conditions Along São Francisco \\ River, Minas Gerais, Brazil \\ MACEDO, J.F. ${ }^{2}$, BRANDÃO, M. ${ }^{3}$ e LARA, J.F.R. ${ }^{4}$
}

\begin{abstract}
RESUMO - Realizou-se um estudo na região do Alto São Francisco, Minas Gerais, no final da safra agrícola 1997/1998, visando identificar as plantas daninhas que permanecem nas áreas após a colheita do milho e sua distribuição ao longo da área estudada, destacando-se as mais importantes. As observações foram realizadas em 12 municípios. Em cada local foi lançado, por cem vezes, um quadrado de $0,50 \times 0,50 \mathrm{~m}$, a espaços de $10 \mathrm{~m}$. Dentro do quadrado foram contadas as espécies e registrado o número de indivíduos de cada uma delas. Foram encontradas 151 espécies em 35 famílias, sendo Asteraceae a mais bem representada, com 25 espécies. As espécies com maior Îndice de Valor de Importância (IVI) foram: Ageratum conyzoides, Sida glaziovii, Conyza bonariensis, Gaya sp., Sida rhombifolia e Blainvillea biaristata. As plantas daninhas remanescentes nas áreas recém-colhidas são fonte de alimento para insetos polinizadores e inimigos naturais das pragas; adequadamente manejadas, elas podem favorecer a manutenção do equilíbrio nos agroecossistemas.
\end{abstract}

Palavras-chave: agricultura sustentável, manejo integrado, plantio direto.

\begin{abstract}
A survey was carried out on the weed populations occurring after harvest in cornfields along the São Francisco River to identify which weeds remained in the 12 municipalities spread along the river, from its headwaters down to "Tres Marias". Ageratum conyzoides $L$. was the only species found in all the fields surveyed. The species presenting major Importance Value Index (IVI) were: Sida glasiovi K. Schum, Conyza bonariensis (L.) Cronq, Gaya sp. Sida rhombifolia $L$. and Blainvillea biaristata DC., being also found in one or more places. These plants are sources of food for certain pollinating and pest predating insects. Such surveys may help to adequately choose herbicides to clean up the fields after corn harvest, avoiding improper applications of chemical products, which represent great risks of environmental pollution.
\end{abstract}

Key words: sustainable agriculture, integrated management, and no-tillage system.

\section{INTRODUÇÃO}

Conhecer as espécies daninhas que ocorrem numa área agrícola é imprescindível para que se possa sugerir o seu manejo e controle seletivo, principalmente no caso de implantação de sistemas de plantio direto, evitando aplicação intensiva de herbicidas em doses desnecessárias. Deve-se considerar que espécies de plantas daninhas, se mantidas em locais estratégicos, como beiradas de cercas, margens de estradas, voçorocas, podem ser extremamente benéficas (Macedo \& Martins, 1998).

A composição das populações de plantas daninhas em um agroecossistema é reflexo de suas características edáficas e climáticas e das práticas agronômicas adotadas, como manejo

1 Recebido para publicação em 24.5.2002 e na forma revisada em 11.8.2003.

Apoio financeiro da Fundação de Amparo à Pesquisa do Estado de Minas Gerais - FAPEMIG.

${ }^{2}$ Biólogo, EPAMIG, Av. José Cândido da Silveira 1647, União, 30170-000 Belo Horizonte-MG; ${ }^{3}$ Taxonomista, EPAMIG;

${ }^{4}$ Biólogo, EPAMIG. 
de solo e aplicação de herbicidas (Godoy et al., 1995; Voll et al., 2001). Assim, o plantio direto pode alterar a população de plantas daninhas, a dinâmica do banco de sementes do solo e a eficiência de herbicidas aplicados em préemergência, devido à cobertura do solo com resíduos vegetais (Crutchfield et al., 1986; Johnson et al., 1989; Bulher et al., 1995).

A Região do Alto São Francisco tem na agropecuária sua atividade econômica mais importante. Após a inclusão do cerrado no processo produtivo, a região tornou-se grande produtora de grãos, principalmente milho e feijão.

Nos solos de várzeas, o cultivo convencional contribui para a degradação do agroecossistema, especialmente pela compactação dos solos e infestação com plantas daninhas. Por essas razões, deve-se voltar a atenção para o sistema de Plantio Direto na Palha, uma das alternativas para melhorar e manter as características físicas e químicas dos solos e preservar sua capacidade produtiva. Além disso, o preparo convencional do solo incorpora as sementes de modo mais uniforme no perfil trabalhado, dando origem a persistentes bancos de sementes no solo (Guersa \& MartinezGuersa, 2000).

Diferentes sistemas de manejo do solo condicionam as sementes a microambientes, devido a alterações das propriedades físicoquímicas e nas condições da superfície do solo (Mulugueta \& Stoltemberg, 1997); quando se usam intensivamente herbicidas não-seletivos, pode ocorrer a seleção de espécies de plantas daninhas tolerantes. Para evitar isso e obter melhor eficiência de controle das plantas daninhas, recomenda-se a formulação de misturas de herbicidas de diferentes mecanismos de ação e que apresentem efeitos sinergísticos ou aditivos (Monquero et al., 2001).

Segundo Pitelli (2000), os índices fitossociológicos são importantes para analisar o impacto que os sistemas de manejo e as práticas agrícolas exercem sobre a dinâmica de crescimento e ocupação de comunidades infestantes em agroecossistemas. Esses índices são determinados levando-se em conta a densidade relativa, que reflete a participação numérica de indivíduos de uma determinada espécie na comunidade; a freqüência relativa, que se refere à porcentagem que representa a freqüência de uma população em relação à soma das freqüências das espécies que constituem a comunidade; a dominância relativa, que representa o ganho de biomassa de uma espécie na comunidade; e a importância relativa, que é uma avaliação ponderada desses índices e indica as espécies mais importantes em termos de infestação das culturas e, também, qual espécie proporciona melhor cobertura do solo no caso de utilizá-la nos sistemas de plantio direto.

Quatro passos podem ser considerados fundamentais para o sucesso do plantio direto: eliminação/redução do preparo do solo, uso de herbicidas dessecantes, obtenção de cobertura morta e uso de plantadeiras específicas. Os três primeiros estão diretamente associados ao manejo de plantas daninhas; por isso, o conhecimento das espécies que permanecem após a colheita de uma cultura é de grande importância, pois estas farão parte da cobertura morta para a cultura subseqüente, o que é fundamental para o sucesso na implantação de sistemas de plantio direto.

Este estudo teve por objetivos conhecer a flora daninha que permanece nas áreas após a colheita do milho e mostrar quais são as principais plantas daninhas ocorrentes nas várzeas do Rio São Francisco, em Minas Gerais, e sua fitossociologia e distribuição ao longo da área estudada.

\section{MATERIAL E MÉTODOS}

Após a colheita do milho, safra agrícola 1997/98, coletaram-se plantas daninhas em 12 municípios do Estado de Minas Gerais banhados pelo Rio São Francisco, a montante da represa de Três Marias. Um quadrado de $50 \times 50 \mathrm{~cm}$ foi jogado cem vezes a intervalos de 10 metros um do outro. Foram anotadas as espécies de plantas daninhas dentro dos limites do quadrado em cada ponto e contado o número de indivíduos de cada uma delas. Quando uma planta não podia ser identificada no campo, sua amostra era registrada sob um número, para posterior identificação.

Amostras das plantas foram coletadas para incorporação ao herbário da Empresa de Pesquisa Agropecuária de Minas Gerais PAMG/EPAMIG. 
A freqüência (Fre), densidade (Den), abundância $(\mathrm{Abu})$, freqüência relativa (Frr), densidade relativa (Der), abundância relativa (Abr) e o índice de valor de importância (IVI) foram calculados utilizando-se as seguintes fórmulas:

Fre $=\frac{\text { Número de parcelas que contêm a espécie }}{\text { Número total de parcelas utilizadas }}$

Den $=\frac{\text { Número total de indivíduos por espécie }}{\text { Área total coletada }}$

$\mathrm{Abu}=\frac{\text { Número total de indivíduos por espécie }}{\text { Número total de parcelas que contêm a espécie }}$

Frr $=\frac{\text { Freqüência da espécie } \times 100}{\text { Freqüência total de todas as espécies }}$

Der $=\frac{\text { Densidade da espécie } \times 100}{\text { Densidade total de todas as espécies }}$

$\mathrm{Abr}=\frac{\text { Abundância da espécie } \times 100}{\text { Abundância total de todas as espécies }}$

$\mathrm{IVI}=F r r+D e r+A b r$

Calculou-se o "Índice de Similaridade de Sorensen" (S) entre as áreas, utilizando a seguinte fórmula: $\mathrm{S}=2 \mathrm{a} / \mathrm{b}+\mathrm{c}$, em que: $\mathrm{a}=$ número de espécies comuns às duas áreas; $\mathrm{b}=$ número total de espécies na área 1; e $\mathrm{c}=$ número total de espécies na área 2. Este índice permite comparar as áreas duas a duas quanto à composição florística, possibilitando recomendar o uso de herbicidas específicos para cada uma delas.

\section{RESULTADOS E DISCUSSÃO}

Foram coletadas, nos 12 municípios estudados, 151 espécies pertencentes a 95 gêneros, agrupados em 33 famílias (Tabela 1).

Ageratum conyzoides foi a única espécie comum a todos os locais. Outras com maior distribuição foram: Emilia sonchifolia e Hyptis suaveolens, encontradas em 11 localidades; e Bidens pilosa, Chamaesyce hirta e Ipomoea grandifolia, encontradas em nove locais.

Na Tabela 2 estão listadas as espécies mais importantes de cada local, escolhidas por ordem decrescente do índice de valor de importância (IVI). Destacaram-se as seguintes espécies: Ageratum conyzoides, em Piumhi, Doresópolis, Bambuí, Iguatama, Martinho Campos e Dores do Indaiá; Sida glaziovii, em Vargem Bonita; Blainvillea biaristata, em Lagoa da Prata; Gaya sp., em Bom Despacho; Conyza bonariensis, em luz; Sida glasiovii, em Pompéu; e Sida rhombifolia, em Abaeté. Essa variação espacial da dominação das plantas infestantes observada neste estudo pode ser reflexo de diferentes sistemas de manejo do solo e de práticas culturais, empregados pelos agricultores em cada local, pois estes fatores influenciam a dinâmica das populações de plantas daninhas (Oryokot et al., 1997).

Tabela 1 - Relação das plantas invasoras encontradas na cultura de milho, nas várzeas do Rio São Francisco, nos seguintes municípios: Vargem Bonita (1), Piumhi (2), Doresópolis (3), Bambuí (4), Iguatama (5), Lagoa da Prata (6), Luz (7), Dores do Indaiá (8), Bom Despacho (9), Martinho Campos (10), Pompéu (11) e Abaeté (12)

\begin{tabular}{|c|c|c|c|c|c|c|c|c|c|c|c|c|c|}
\hline \multirow{2}{*}{ Espécie } & \multirow{2}{*}{ Família } & \multicolumn{12}{|c|}{ Município de Ocorrência } \\
\hline & & 1 & 2 & 3 & 4 & 5 & 6 & 7 & 8 & 9 & 10 & 11 & 12 \\
\hline Alternanthera tenella Colla & Amaranthaceae & & & $\mathrm{x}$ & $\mathrm{x}$ & $\mathrm{x}$ & & & & $\mathrm{x}$ & & & $\mathrm{x}$ \\
\hline Acanthospermum australe (Loef) Kunt & Asteraceae & $\mathrm{x}$ & & & $\mathrm{x}$ & & & & $\mathrm{x}$ & $\mathrm{x}$ & $\mathrm{x}$ & $\mathrm{x}$ & $\mathrm{x}$ \\
\hline Acanthospermum hispidum DC. & Asteraceae & & & & & & & & & & & & $\mathrm{x}$ \\
\hline Ageratum conyzoides L. & Asteraceae & $\mathrm{x}$ & $\mathrm{x}$ & $\mathrm{x}$ & $\mathrm{x}$ & $\mathrm{x}$ & $\mathrm{x}$ & $\mathrm{x}$ & $\mathrm{x}$ & $\mathrm{x}$ & $\mathrm{x}$ & $\mathrm{x}$ & $\mathrm{x}$ \\
\hline Aeschynomene falcata (Poir) DC. & Leguminosae - Pap. & & & & & & & & & & & & $\mathrm{x}$ \\
\hline Amaranthus blitum L. & Amaranthaceae & & & & & $\mathrm{x}$ & & $\mathrm{x}$ & & & & & \\
\hline Amaranthus deflexus L. & Amaranthaceae & & & & & & & $\mathrm{x}$ & & & & & \\
\hline Amaranthus lividus L. & Amaranthaceae & & & & & $\mathrm{x}$ & & & & & & & \\
\hline Amaranthus retroflexus L. & Amaranthaceae & & $x$ & & & & & & & & & & \\
\hline Amaranthus spinosus L. & Amaranthaceae & & $\mathrm{x}$ & & & & & & & & & & $\mathrm{x}$ \\
\hline
\end{tabular}

Continua... 
Tabela 1, cont.

\begin{tabular}{|c|c|c|c|c|c|c|c|c|c|c|c|c|c|}
\hline \multirow{2}{*}{ Espécie } & \multirow{2}{*}{ Família } & \multicolumn{12}{|c|}{ Município de Ocorrência } \\
\hline & & 1 & 2 & 3 & 4 & 5 & 6 & 7 & 8 & 9 & 10 & 11 & 12 \\
\hline Amaranthus viridis $\mathrm{L}$. & Amaranthaceae & $\mathrm{x}$ & $\mathrm{x}$ & & & & $\mathrm{x}$ & & & & & & \\
\hline Apium leptophyllum (Pers.) Muell-Arg & Apiaceae & & & & & & & $\mathrm{x}$ & & & & & \\
\hline Arachis sp. & Leguminosae - Pap. & $\mathrm{x}$ & & & & & & & & & & & \\
\hline Asclepias curassavica $\mathrm{L}$. & Asclepiadaceae & & & & & & & $\mathrm{x}$ & & & & & $\mathrm{x}$ \\
\hline Baccharis dracunculifolia DC. & Asteraceae & & & & & & & & & $\mathrm{x}$ & & & \\
\hline Bidens pilosa $\mathrm{L}$. & Asteraceae & & & $\mathrm{x}$ & $x$ & $\mathrm{x}$ & $\mathrm{x}$ & $\mathrm{x}$ & $\mathrm{x}$ & $\mathrm{x}$ & & $\mathrm{x}$ & $\mathrm{x}$ \\
\hline Blainvillea biaristata DC. & Asteraceae & & & & & $x$ & $x$ & & & & & & $\mathrm{x}$ \\
\hline Blainvillea rhomboidea Cass. & Asteraceae & $x$ & & & $x$ & 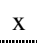 & 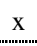 & $\mathrm{x}$ & & $\mathrm{x}$ & $x$ & & $x$ \\
\hline Brachiaria decumbens Stapf. & Poaceae & $x$ & $x$ & & & & $\mathrm{x}$ & & & & & & \\
\hline Brachiaria mutica (Forsk.) Stapf. & Poaceae & & & & & $\mathrm{x}$ & & & & & & & $\mathrm{x}$ \\
\hline Brachiaria ruziziensis Germ. \& Evrard & Poaceae & $\mathrm{x}$ & $x$ & & & & & & & & $\mathrm{x}$ & & $\mathrm{x}$ \\
\hline Buddleja brasiliensis Jacq. & Buddlejaceae & & & & & $\mathrm{x}$ & & & & & & & \\
\hline Calopogonium mucunoides Desv. & Leguminosae - Pap. & & & & & & & $\mathrm{x}$ & & & & $\mathrm{x}$ & \\
\hline Canna indica $\mathrm{L}$. & Cannaceae & & & & & & & $\mathrm{x}$ & & & & $\mathrm{x}$ & \\
\hline Caperonia palustris (L.) St. Hil. & Euphorbiaceae & $\mathrm{x}$ & & & & & & & & & & & \\
\hline Cardiospermum halicacabum L. & Sapindaceae & & & & & & & $\mathrm{x}$ & & & & & \\
\hline Cenchrus echinatus $\mathrm{L}$. & Poaceae & $\mathrm{x}$ & & & & & & & & & & & \\
\hline Centratherum punctatum Cass. & Asteraceae & & $x$ & & & & & & & & & & \\
\hline Chamaecrista patellaria Green. & Leguminosae-Caes. & $\mathrm{x}$ & & & & & & & & & & & \\
\hline Chamaecrista rotundifolia (Per.) Gree. & Leguminosae-Caes. & & & & & & $x$ & & & & & & \\
\hline Chamaesyce hirta (L.) Mill. & Euphorbiaceae & & & $\mathrm{x}$ & $\mathrm{x}$ & $\mathrm{x}$ & $\mathrm{x}$ & $\mathrm{x}$ & $\mathrm{x}$ & $\mathrm{x}$ & $\mathrm{x}$ & $\mathrm{x}$ & $\mathrm{x}$ \\
\hline Chamaesyce hyssopifolia (L.) Small & Euphorbiaceae & $x$ & & & & & & & $\mathrm{x}$ & & $\mathrm{x}$ & & $\mathrm{x}$ \\
\hline Chaptalia integerrima (Vell.) Burk. & Asteraceae & & $x$ & & & & & & & & & & \\
\hline Chenopodium ambrosioides L. & Chenopodiaceae & & & & & & & & $\mathrm{x}$ & & & & \\
\hline Chloris sp. & Poaceae & & & & & $x$ & & & & & & & \\
\hline Cleome spinosa $\mathrm{L}$. & Capparaceae & & & & & & & & & & & $x$ & \\
\hline Commelina benghalensis L. & Commelinaceae & & & & $x$ & $x$ & $x$ & $x$ & $x$ & & & & $\mathrm{x}$ \\
\hline Conyza bonariensis (L.) Cronq. & Asteraceae & & $x$ & & $x$ & & & $\mathrm{x}$ & $x$ & $\mathrm{x}$ & & $\mathrm{x}$ & \\
\hline Corchorus hirtus L. & Tiliaceae & & & $\mathrm{x}$ & $\mathrm{x}$ & & $\mathrm{x}$ & $\mathrm{x}$ & $\mathrm{x}$ & $\mathrm{x}$ & & $\mathrm{x}$ & $\mathrm{x}$ \\
\hline Cordia verbenaceae DC. & Boraginaceae & & & & & & & & & $\mathrm{x}$ & & & \\
\hline Crotalaria anagiroides H.B.K. & Leguminosae - Pap. & & & & & & $\mathrm{x}$ & & & & & & \\
\hline Crotalaria incana $\mathrm{L}$. & Leguminosae - Pap. & & & & & & & & & $\mathrm{x}$ & & & \\
\hline Crotalaria lanceolata E. Mey & Leguminosae - Pap. & & & & & & & & & & & & $\mathrm{x}$ \\
\hline Croton grandulosus L. & Euphorbiaceae & $x$ & & & & & & & & & & & \\
\hline Croton urucurana Bail. & Euphorbiaceae & & & & & & & & $\mathrm{x}$ & $\mathrm{x}$ & & & \\
\hline Cucumis anguria $\mathrm{L}$. & Cucurbitaceae & & & & & & & & $\mathrm{x}$ & & & & \\
\hline Cuphea carthaginensis (Jacq.) Macbr. & Lythraceae & $\mathrm{x}$ & & & & & & & & & $x$ & & $\mathrm{x}$ \\
\hline Cynodon dactylon (L.) Pers. & Poaceae & & & & & & & & & $\mathrm{x}$ & & & $\mathrm{x}$ \\
\hline Cyperus rotundus L. & Cyperaceae & $x$ & & & & $x$ & & & & $\mathrm{x}$ & $x$ & $x$ & \\
\hline Desmodium barbatum (L.) Benth. & Leguminosae - Pap. & $\mathrm{x}$ & $x$ & & & & & & & & $\mathrm{x}$ & $\mathrm{x}$ & \\
\hline Desmodium incanum (G.) Sch. et Thel. & Leguminosae - Pap. & $\mathrm{x}$ & & $x$ & & & & & $\mathrm{x}$ & & & $\mathrm{x}$ & $\mathrm{x}$ \\
\hline Dichondra repens Forst. & Convolvulaceae & & & & & & & & $\mathrm{x}$ & & & $\mathrm{x}$ & $\mathrm{x}$ \\
\hline Digitaria horizontalis Willd. & Poaceae & & & & & & & $\mathrm{x}$ & & & & $x$ & \\
\hline Digitaria sanguinalis (L.) Scop. & Poaceae & $\mathrm{x}$ & & & & & & $\mathrm{x}$ & & & & $\mathrm{x}$ & \\
\hline Echinochloa cruzgali (L.) Beauv. & Poaceae & & & & & $x$ & & & & & & $\mathrm{x}$ & \\
\hline Eclipta alba Hassk. & Asteraceae & & & & $\mathrm{x}$ & & & $\mathrm{x}$ & $\mathrm{x}$ & $\mathrm{x}$ & $\mathrm{x}$ & $\mathrm{x}$ & $\mathrm{x}$ \\
\hline Elephantopus mollis H. B. K. & Asteraceae & & & & & & & & $x$ & & & & $\mathrm{x}$ \\
\hline
\end{tabular}


Tabela 1, cont.

\begin{tabular}{|c|c|c|c|c|c|c|c|c|c|c|c|c|c|}
\hline \multirow{2}{*}{ Espécie } & \multirow{2}{*}{ Família } & \multicolumn{12}{|c|}{ Município de Ocorrência } \\
\hline & & 1 & 2 & 3 & 4 & 5 & 6 & 7 & 8 & 9 & 10 & 11 & 12 \\
\hline Eleusine indica (L.) Gaertn. & Poaceae & & $\mathrm{x}$ & & & $\mathrm{x}$ & & & & & $\mathrm{x}$ & $\mathrm{x}$ & $\mathrm{x}$ \\
\hline Elvira biflora (L.) DC. & Asteraceae & & & $\mathrm{x}$ & $\mathrm{x}$ & & $\mathrm{x}$ & & $\mathrm{x}$ & & & $\mathrm{x}$ & \\
\hline Emilia sonchifolia DC. & Asteraceae & $\mathrm{x}$ & & $\mathrm{x}$ & $x$ & $x$ & $\mathrm{x}$ & $\mathrm{x}$ & $\mathrm{x}$ & $\mathrm{x}$ & $\mathrm{x}$ & $\mathrm{x}$ & $\mathrm{x}$ \\
\hline Eragrostes sp. & Poaceae & & & & & & & & & $x$ & & & \\
\hline Eupatorium pauciflorum H. B. K. & Asteraceae & & & & & & $\mathrm{x}$ & $\mathrm{x}$ & $\mathrm{x}$ & & $\mathrm{x}$ & & \\
\hline Euphorbia heterophylla L. & Euphorbiaceae & $x$ & & & & $x$ & $x$ & $\mathrm{x}$ & $\mathrm{x}$ & & & & \\
\hline Galinsoga parviflora Cav. & Asteraceae & & & & & & & $x$ & & $\mathrm{x}$ & $\mathrm{x}$ & $\mathrm{x}$ & \\
\hline Gaya sp. & Malvaceae & & & & & & & & & $x$ & & & \\
\hline Heliotropium indicum L. & Boraginaceae & & & & & $x$ & & & $\mathrm{x}$ & & $\mathrm{x}$ & & $\mathrm{x}$ \\
\hline Hyparrhenia rufa (Nees) Stapf & Poaceae & & & $\mathrm{x}$ & & & & & & & & $\mathrm{x}$ & \\
\hline Hyptis lophantha Mart. ex Benth. & Lamiaceae & & & $\mathrm{x}$ & $\mathrm{x}$ & $\mathrm{x}$ & & $\mathrm{x}$ & $\mathrm{x}$ & $\mathrm{x}$ & & & $\mathrm{x}$ \\
\hline Hyptis densiflora Mart. & Lamiaceae & & & $\mathrm{x}$ & & & & $\mathrm{x}$ & & & & $\mathrm{x}$ & $\mathrm{x}$ \\
\hline Hyptis suaveolens (L.) Poit. & Lamiaceae & & $x$ & $x$ & $\mathrm{x}$ & $x$ & $x$ & $\mathrm{x}$ & $\mathrm{x}$ & $x$ & $\mathrm{x}$ & $\mathrm{x}$ & $\mathrm{x}$ \\
\hline Indigofera suffruticosa Mill. Gardn. & Lguminosae - Pap. & & & & & & & $\mathrm{x}$ & & & & & \\
\hline Ipomoea cairica $(\mathrm{L}.) \mathrm{Sw}$. & Convolvulaceae & & & & & & $\mathrm{x}$ & & & & & $\mathrm{x}$ & \\
\hline Ipomoea grandifolia (Damm.) O' Don. & Convolvulaceae & $\mathrm{x}$ & & $x$ & $\mathrm{x}$ & $\mathrm{x}$ & $\mathrm{x}$ & $\mathrm{x}$ & & $x$ & $\mathrm{x}$ & $\mathrm{x}$ & $\mathrm{x}$ \\
\hline Ipomoea hederifolia $\mathrm{L}$. & Convolvulaceae & & & $\mathrm{x}$ & & $\mathrm{x}$ & & & & & & & \\
\hline Ipomoea purpurea (L.) Roth. & Convolvulaceae & & & & & $\mathrm{x}$ & & & & & & & \\
\hline Ipomoea quamoclit $\mathrm{L}$. & Convolvulaceae & & & & $x$ & & & & & & & & \\
\hline Ipomoea São paulista & Convolvulaceae & $x$ & & $\mathrm{x}$ & & & & & & & & & \\
\hline Lantana camara L. & $\begin{array}{l}\text { Verbenaceae } \\
\text { Verbeacen }\end{array}$ & & & & & & & & $\mathrm{x}$ & $\mathrm{x}$ & & & \\
\hline Leonotis nepetifolia (L.) W.T.Aiton & Lamiaceae & & & $\mathrm{x}$ & $\mathrm{x}$ & $\mathrm{x}$ & & $\mathrm{x}$ & $\mathrm{x}$ & $\mathrm{x}$ & & $\mathrm{x}$ & $\mathrm{x}$ \\
\hline Leonurus sibiricus $\mathrm{L}$. & Lamiaceae & & $x$ & & & $x$ & & $\mathrm{x}$ & $\mathrm{x}$ & & & & \\
\hline Lepidium virginicum $\mathrm{L}$. & $\begin{array}{l}\text { Brassicaceae } \\
\text { Bracis. }\end{array}$ & & & & & $x$ & & $\mathrm{x}$ & & & & & \\
\hline Malvastrum americanum (L.) Torr. & Malvacee & & & & & $\mathrm{x}$ & & & & & & & \\
\hline Malvastrum coromandelianum (L.) Ga. & Malvacee & & & & & & & & & $\mathrm{x}$ & & $\mathrm{x}$ & \\
\hline Manetia ignita Schum. & Rubiaceae & & & & & & & $\mathrm{x}$ & & & & & $\mathrm{x}$ \\
\hline Marsypianthes chamaedrys (V.) Kunt. & Lamiacee & & & & & & & & & $x$ & $\mathrm{x}$ & & \\
\hline Momordica charantia $\mathrm{L}$. & Cucurbitaceae & & & & & & & & & & & $\mathrm{x}$ & \\
\hline Nicandra physaloides (L.) Pers. & Solanaceae & & & & & $\mathrm{x}$ & & & & & & & \\
\hline Oxalis corniculata $\mathrm{L}$. & Oxalidaceae & & & & & $x$ & & & $\mathrm{x}$ & $\mathrm{x}$ & & & \\
\hline Panicum maximum Jacq. & Poaceae & & & & & $\mathrm{x}$ & $\mathrm{x}$ & $\mathrm{x}$ & & & & & \\
\hline Paspalum notatum Fliigge & Poaceae & $\mathrm{x}$ & & & & & & & $\mathrm{x}$ & & $\mathrm{x}$ & $\mathrm{x}$ & \\
\hline Pavonia cancellata Cav. & Malvaceae & & & & & $\mathrm{x}$ & & & & & & & \\
\hline Phillanthus niruri L. & Euphorbiaceae & & & & & & $x$ & & & & & & \\
\hline Phyllanthus tenellus Roxb. & Euphorbiaceae & & & $\mathrm{x}$ & $\mathrm{x}$ & $\mathrm{x}$ & & & $\mathrm{x}$ & & $\mathrm{x}$ & & $\mathrm{x}$ \\
\hline Physalis angulata $\mathrm{L}$. & Solanaceae & $\mathrm{x}$ & $\mathrm{x}$ & & $\mathrm{x}$ & $\mathrm{x}$ & & & $\mathrm{x}$ & & $\mathrm{x}$ & $\mathrm{x}$ & \\
\hline Polygala lancifolia St. Hil. & Polygalaceae & & & & & & $x$ & & & $\mathrm{x}$ & & & \\
\hline Polygala paniculata $\mathrm{L}$. & Polygalaceae & $x$ & & $x$ & $x$ & & & & & & & & \\
\hline Porophyllum ruderale (Jacq.) Cass. & Asteraceae & & & & & & $x$ & & $\mathrm{x}$ & & $\mathrm{x}$ & $\mathrm{x}$ & $\mathrm{x}$ \\
\hline Portulaca oleracea $\mathrm{L}$. & Portulacaceae & $\mathrm{x}$ & & & & & & & & & & & \\
\hline Psidium guajava $\mathrm{L}$. & Myrtaceae & & & & & & & & $\mathrm{x}$ & $\mathrm{x}$ & $\mathrm{x}$ & & \\
\hline Pyrostegia venusta (Ker Gawl) Miers. & Bignoniaceae & & & $x$ & & & $x$ & & & & & & \\
\hline Rhynchosia minima DC. & Leguminosae - Pap. & & & & & & $\mathrm{x}$ & & & & & & \\
\hline Richardia brasiliensis Gómez & Rubiaceae & & $x$ & & $\mathrm{x}$ & & $\mathrm{x}$ & & & & & & $\mathrm{x}$ \\
\hline Richardia scabra $\mathrm{L}$. & Rubiaceae & & & & & & & $\mathrm{x}$ & & & & & \\
\hline Ricinus communis $\mathrm{L}$. & Euphorbiaceae & & & & $\mathrm{x}$ & & & $\mathrm{x}$ & & & & & \\
\hline
\end{tabular}

Continua... 
Tabela 1, cont.

\begin{tabular}{|c|c|c|c|c|c|c|c|c|c|c|c|c|c|}
\hline \multirow{2}{*}{ Espécie } & \multirow{2}{*}{ Família } & \multicolumn{12}{|c|}{ Município de Ocorrência } \\
\hline & & 1 & 2 & 3 & 4 & 5 & 6 & 7 & 8 & 9 & 10 & 11 & 12 \\
\hline Scoparia dulcis $\mathrm{L}$. & Scrophulariaceae & & & & & & & & $\mathrm{x}$ & $\mathrm{x}$ & $\mathrm{x}$ & $\mathrm{x}$ & $\mathrm{x}$ \\
\hline Senna alata (L.) Roxb. & Leguminosae- Caes. & & & & $\mathrm{x}$ & & & & & & & & \\
\hline Senna obtusifolia (L.) Irwin \& Barn. & Leguminosae- Caes. & $\mathrm{x}$ & & & $\mathrm{x}$ & & $\mathrm{x}$ & & & $\mathrm{x}$ & & $\mathrm{x}$ & $\mathrm{x}$ \\
\hline Senna occidentalis (L.) Link. & Leguminosae- Caes. & & $\mathrm{x}$ & $x$ & & $\mathrm{x}$ & & $\mathrm{x}$ & & & & & \\
\hline Sida carpinifolia L.f. & Malvaceae & & & & & $x$ & $x$ & & & $\mathrm{X}$ & $x$ & $x$ & \\
\hline Sida cerradoensis Krapov. & Malvaceae & & & & & & & & & & $\mathrm{x}$ & & \\
\hline Sida cordifolia $\mathrm{L}$. & Malvaceae & & & & & $x$ & $\mathrm{x}$ & $\mathrm{x}$ & $\mathrm{x}$ & & $\mathrm{x}$ & & $\mathrm{x}$ \\
\hline Sida glaziovii K. Schum. & Malvaceae & $\mathrm{x}$ & & $\mathrm{x}$ & & & $\mathrm{x}$ & $x$ & $\mathrm{x}$ & $\mathrm{x}$ & $\mathrm{x}$ & $\mathrm{x}$ & $\mathrm{x}$ \\
\hline Sida linifolia Cav. & Malvaceae & $x$ & & $x$ & & & & & & & $x$ & $x$ & \\
\hline Sida rhombifolia $\mathrm{L}$. & Malvaceae & $\mathrm{x}$ & $\mathrm{x}$ & & & $\mathrm{x}$ & $\mathrm{x}$ & $\mathrm{x}$ & & & $\mathrm{x}$ & $\mathrm{x}$ & $\mathrm{x}$ \\
\hline Sida santaremnensis Monteiro & Malvaceae & & & & & $\mathrm{X}$ & & & & $\mathrm{X}$ & & & \\
\hline Sida spinosa $\mathrm{L}$. & Malvaceae & & & $x$ & $\mathrm{x}$ & & & $\mathrm{x}$ & & & & & $\mathrm{X}$ \\
\hline Sida urens L. & Malvaceae & $\mathrm{x}$ & & $x$ & & $x$ & $\mathrm{x}$ & $x$ & $\mathrm{x}$ & $\mathrm{x}$ & $\mathrm{x}$ & $\mathrm{x}$ & $\mathrm{x}$ \\
\hline Sida viarum St. Hil. & Malvaceae & & & & & & & $x$ & $x$ & $\mathrm{x}$ & & & $\mathrm{x}$ \\
\hline Sidastrum micranthum (St Hil.) Fry. & Malvaceae & & & & & & & & $\mathrm{x}$ & & & & $\mathrm{x}$ \\
\hline Solanum aculeatissimum Jacq. & Solanaceae & $\mathrm{x}$ & & & & & & $\mathrm{X}$ & $\mathrm{x}$ & & & $\mathrm{x}$ & \\
\hline Solanum americanum Mill. & Solanaceae & $\mathrm{x}$ & $\mathrm{x}$ & & & & & & $\mathrm{x}$ & & & $\mathrm{x}$ & $\mathrm{X}$ \\
\hline Solanum lycocarpum St. Hil. & Solanaceae & $\mathrm{x}$ & & & & & & & $\mathrm{x}$ & & & $\mathrm{x}$ & $\mathrm{X}$ \\
\hline Solanum paniculatum L. & Solanaceae & & & - & & & & & $\mathrm{x}$ & $x$ & & & $\mathrm{x}$ \\
\hline Solanum sisymbriifolium Lam. & Solanaceae & & & & & & & & & & & & $\mathrm{x}$ \\
\hline Sonchus oleraceus L. & Asteraceae & & & & $x$ & $x$ & $\mathrm{x}$ & $x$ & $\mathrm{x}$ & & & & \\
\hline Sorghum arundinaceum (Willd.) Stapf & Poaceae & & & & $\mathrm{x}$ & & & & & & & $\mathrm{x}$ & \\
\hline Spermacaoce alata Aubl. & Rubiaceae & $x$ & & & & & & & $x$ & & & & \\
\hline Spermacoce hirta L. & Rubiaceae & & $\mathrm{X}$ & & & & & & & & & & \\
\hline Spermacoce hyssopifoia (L.) Small. & Rubiaceae & & & $x$ & & & & & & & & & \\
\hline Spermacoce latifolia Aubl. & Rubiaceae & $\mathrm{x}$ & & $\mathrm{X}$ & & & & & & & & & \\
\hline Spermacoce verticilata $\mathrm{L}$. & Rubiaceae & $\mathrm{x}$ & $\mathrm{x}$ & $\mathrm{x}$ & $\mathrm{x}$ & & & & & & $\mathrm{x}$ & & \\
\hline Spilanthes acmella (L.) Murray & Asteraceae & & & & & & & & & & & $x$ & \\
\hline Stachytarpheta cayennensis (Rich.) Vahl.' & Verbenaceae & $\mathrm{x}$ & & "'m & & & X & " & $\mathrm{x}$ & $\mathrm{X}$ & $\mathrm{x}$ & & $\mathrm{x}$ \\
\hline Stylosanthes acuminata M.B.Fer. et Costa & Leguminosae - Pap. & & & & & & & & & & $\mathrm{x}$ & & \\
\hline Stylosanthes grandifolia M.B.Fer. et Costa & Leguminosae - Pap. & $\mathrm{x}$ & & & & & & & $\mathrm{X}$ & & & & \\
\hline Stylosanthes guyanensis (Aubl.) Sw. & Leguminosae - Pap. & $\mathrm{X}$ & & & & & $\mathrm{X}$ & & & & & & $\mathrm{X}$ \\
\hline Stylosanthes scabra Vog. & Leguminosae - Pap. & & & & & & & $\mathrm{X}$ & & & & $x$ & \\
\hline Tagetes minuta $\mathrm{L}$. & Asteraceae & & & $x$ & & & $\mathrm{x}$ & & & & & & \\
\hline Tallinum paniculatum (Jacq.) Gaertn. & Portulacaceae & & & & & $x$ & & & & & & & \\
\hline Tridax procumbens L. & Asteraceae & & & & & & & $x$ & & $\mathrm{X}$ & & & \\
\hline Triumfetta semitriloba Jacq. & Tiliaceae & & & $\mathrm{x}$ & & & & & $\mathrm{x}$ & $\mathrm{X}$ & $\mathrm{x}$ & & $\mathrm{x}$ \\
\hline Triumfetta bartramia L. & Tiliaceae & $x$ & & $x$ & & & $\mathrm{x}$ & $x$ & & & & & \\
\hline Turnera ulmifolia L & Turneraceae & & & $\mathrm{x}$ & & & & $\mathrm{x}$ & $\mathrm{x}$ & & & & \\
\hline Urena lobata L. & Malvaceae & & & & & & & & & & & & $x$ \\
\hline Vernonia polyanthes Lees. & Asteraceae & $\mathrm{x}$ & & $\mathrm{x}$ & $\mathrm{x}$ & $\mathrm{x}$ & $\mathrm{X}$ & $\mathrm{x}$ & $\mathrm{X}$ & $\mathrm{X}$ & $\mathrm{x}$ & $\mathrm{x}$ & $\mathrm{X}$ \\
\hline Vernonia remotiflora Rich. & Asteraceae & & & $\mathrm{X}$ & $\mathrm{X}$ & & $\mathrm{X}$ & $x$ & $\mathrm{X}$ & $\mathrm{X}$ & $\mathrm{x}$ & & $\mathrm{X}$ \\
\hline Waltheria americana L. & Sterculiaceae & X & & & & & & $x$ & $\mathrm{x}$ & $\mathrm{x}$ & $\mathrm{x}$ & $\mathrm{x}$ & $\mathrm{X}$ \\
\hline Wedelia pohlii DC. & Asteraceae & & & & & & & & $\mathrm{x}$ & & & & \\
\hline Wissadula subpeltata (Kunt.) Fries & Malvaceae & & & & & & & & & & & $\mathrm{x}$ & $\mathrm{x}$ \\
\hline Zornia latifolia $\mathrm{Sm}$. & Leguminosae - Pap. & & & & & & & & & & & & $\mathrm{X}$ \\
\hline Zornia reticulata $\mathrm{Sm}$. & Leguminosae - Pap. & & & & & & & & & & $\mathrm{x}$ & & \\
\hline
\end{tabular}

Planta Daninha, Viçosa-MG, v.21, n.2, p.239-248, 2003 
Tabela 2 - Relação de freqüência (Fre), densidade (Den), abundância (Abu), frequiência relativa (Frr), densidade relativa (Der), abundância relativa (Abr) e índice de valor de importância (IVI) das plantas invasoras mais importantes em cada município estudado nas margens do Rio São Francisco. ( $\mathrm{N}^{\mathrm{o}}$ Ind. = número total de indivíduos em cada espécie; № Parc. = número de parcelas em que a espécie foi encontrada)

\begin{tabular}{|c|c|c|c|c|c|c|c|c|c|c|}
\hline Local/Espécie & Família & $\begin{array}{c}\mathrm{N}^{\mathrm{o}} \\
\text { Ind. }\end{array}$ & $\begin{array}{c}\mathrm{N}^{\mathrm{o}} \\
\text { Parc. }\end{array}$ & Fre & Den & Abu & Frr & Der & Abr & IVI \\
\hline \multicolumn{11}{|l|}{ Vargem Bonita } \\
\hline Sida glasiovii K Schum. & Malvaceae & 223 & 89 & 0,89 & 8,92 & 2,51 & 19,82 & 27,29 & 3,76 & 50,87 \\
\hline Cyperus rotundus $\mathrm{L}$. & Cyperaceae & 169 & 35 & 0,35 & 6,76 & 4,83 & 7,80 & 20,69 & 7,24 & 35,72 \\
\hline Sida linifolia Cav. & Malvaceae & 57 & 44 & 0,44 & 2,28 & 1,30 & 9,80 & 6,98 & 1,94 & 18,72 \\
\hline Brachiaria decumbens Stapf & Poaceae & 52 & 41 & 0,41 & 2,08 & 1,27 & 9,13 & 6,36 & 1,90 & 17,40 \\
\hline Spermacoce laifolia Aubl. & Rubiaceae & 51 & 41 & 0,41 & 2,04 & 1,24 & 9,13 & 6,24 & 1,86 & 17,24 \\
\hline Arachis sp. & Leguminosae-Pap. & 52 & 36 & 0,36 & 2,08 & 1,44 & 8,02 & 6,36 & 2,17 & 16,55 \\
\hline Spermacoce verticilata L. & Rubiaceae & 52 & 31 & 0,31 & 2,08 & 1,68 & 6,90 & 6,36 & 2,51 & 15,78 \\
\hline Sida rhombifolia L. & Malvaceae & 10 & 2 & 0,02 & 0,40 & 5,00 & 0,45 & 1,22 & 7,49 & 9,16 \\
\hline Ipomoea grandifolia (Dammer) O'don. & Convolvulaceae & 15 & 15 & 0,15 & 0,60 & 1,00 & 3,34 & 1,84 & 1,50 & 6,68 \\
\hline Paspalum notatum Fliigge & Poaceae & 15 & 13 & 0,13 & 0,60 & 1,15 & 2,90 & 1,84 & 1,73 & 6,46 \\
\hline \multicolumn{11}{|l|}{ Piumhi } \\
\hline Ageratum canyzoides $\mathrm{L}$. & Asteraceae & 344 & 92 & 0,92 & 13,76 & 3,74 & 33,33 & 40,95 & 8,36 & 82,64 \\
\hline Conyza bonariensis (L.) Cronq. & Asteraceae & 226 & 17 & 0,17 & 9,04 & 13,29 & 6,16 & 26,90 & 29,71 & 62,77 \\
\hline Brachiaria decumbens Stapf & Poaceae & 185 & 92 & 0,92 & 7,40 & 2,01 & 33,33 & 22,02 & 4,49 & 59,85 \\
\hline Richardia brasiliensis Gómez & Rubiacee & 27 & 22 & 0,22 & 1,08 & 1,23 & 7,97 & 3,21 & 2,74 & 13,93 \\
\hline Hyptis suaveolens (L.) Poit. & Lamiaceae & 18 & 17 & 0,17 & 0,72 & 1,06 & 6,16 & 2,14 & 2,37 & 10,67 \\
\hline Eleusine indica (L.) Gaertn. & Poaceae & 5 & 4 & 0,04 & 0,20 & 1,25 & 1,45 & 0,60 & 2,79 & 4,84 \\
\hline Centratherium punctatum Cass. & Asteraceae & 4 & 3 & 0,03 & 0,16 & 1,33 & 1,09 & 0,48 & 2,98 & 4,54 \\
\hline Chaptalia integerrima (Vell.) Burk. & Asteraceae & 4 & 3 & 0,03 & 0,16 & 1,33 & 1,09 & 0,48 & 2,98 & 4,54 \\
\hline Spermacoce verticillata L. & Rubiaceae & 3 & 2 & 0,02 & 0,12 & 1,50 & 0,72 & 0,36 & 3,35 & 4,43 \\
\hline Amaranthus spinosus L. & Amaranthaceae & 3 & 3 & 0,03 & 0,12 & 1,00 & 1,09 & 0,36 & 2,23 & 3,68 \\
\hline Sida rhombifolia L. var. typica & Malvaceae & 3 & 3 & 0,03 & 0,12 & 1,00 & 1,09 & 0,36 & 2,23 & 3,68 \\
\hline \multicolumn{11}{|l|}{ Doresópolis } \\
\hline Ageratum conyzoides $\mathrm{L}$. & Asteraceae & 1002 & 99 & 0,99 & 40,08 & 10,12 & 24,15 & 70,61 & 14,41 & 109,17 \\
\hline Hyptis suaveolens Poit. & Lamiaceae & 116 & 71 & 0,71 & 4,64 & 1,63 & 17,32 & 8,17 & 2,33 & 27,82 \\
\hline Triunfeta semitriloba Jacq. & Tiliaceae & 77 & 49 & 0,49 & 3,08 & 1,57 & 11,95 & 5,43 & 2,24 & 19,62 \\
\hline Ipomoea hederifolia $\mathrm{L}$. & Convolvulaceae & 38 & 24 & 0,24 & 1,52 & 1,58 & 5,85 & 2,68 & 2,25 & 10,79 \\
\hline Phyllanthus tenellus Roxb. & Euphorbiaceae & 15 & 8 & 0,08 & 0,60 & 1,88 & 1,95 & 1,06 & 2,67 & 5,68 \\
\hline Chamaesyce hirta (L.) Mill. & Euphorbiaceae & 15 & 14 & 0,14 & 0,60 & 1,07 & 3,41 & 1,06 & 1,53 & 6,00 \\
\hline Leonotis nepetifolia (L.) W.T. Aiton & Lamiaceae & 10 & 4 & 0,04 & 0,40 & 2,50 & 0,98 & 0,70 & 3,56 & 5,24 \\
\hline Spermacoce latifolia Aubl. & Rubiaceae & 10 & 6 & 0,06 & 0,40 & 1,67 & 1,46 & 0,70 & 2,37 & 4,54 \\
\hline Elvira biflora (L.) DC. & Asteraceae & 5 & 2 & 0,02 & 0,20 & 2,50 & 0,49 & 0,35 & 3,56 & 4,40 \\
\hline Emilia sonchifolia $L$. & Asteraceae & 11 & 9 & 0,09 & 0,44 & 1,22 & 2,20 & 0,78 & 1,74 & 4,71 \\
\hline \multicolumn{11}{|l|}{ Bambuí } \\
\hline Ageratum conyzoides $\mathrm{L}$. & Asteraceae & 1951 & 99 & 0,99 & 78,04 & 19,71 & 29,64 & 86,25 & 33,03 & 148,92 \\
\hline Hyptis suaveolens Poit. & Lamiaceae & 42 & 32 & 0,32 & 1,68 & 1,31 & 9,58 & 1,86 & 2,20 & 13,6 \\
\hline Vernonia remotiflora Rich & Asteraceae & 36 & 27 & 0,27 & 1,44 & 1,33 & 8,08 & 1,59 & 2,23 & 11,91 \\
\hline Bidens pilosa $\mathrm{L} .$. & Asteraceae & 47 & 18 & 0,18 & 1,88 & 2,61 & 5,39 & 2,08 & 4,38 & 11,8 \\
\hline Chamaessyce hirta (L.) Mill. & Euphorbiaceae & 26 & 20 & 0,20 & 1,04 & 1,30 & 5,99 & 1,15 & 2,18 & 9,32 \\
\hline Spermacoce verticillata $\mathrm{L}$. & Rubiaceae & 20 & 18 & 0,18 & 0,80 & 1,11 & 5,39 & 0,88 & 1,86 & 8,14 \\
\hline Emilia sonchyfolia $\mathrm{L}$. & Asteraceae & 23 & 14 & 0,14 & 0,92 & 1,64 & 4,19 & 1,02 & 2,75 & 7,96 \\
\hline Phyllanthus tenellus Roxb. & Euphorbiaceae & 16 & 10 & 0,10 & 0,64 & 1,60 & 2,99 & 0,71 & 2,68 & 6,38 \\
\hline Ipomoea grandifolia (D.) O'Don & Convolvulaceae & 12 & 12 & 0,12 & 0,48 & 1,00 & 3,59 & 0,53 & 1,68 & 5,80 \\
\hline Corchorus hirtus L. & Tiliaceae & 12 & 11 & 0,11 & 0,48 & 1,09 & 3,29 & 0,53 & 1,83 & 5,65 \\
\hline
\end{tabular}


Tabela 2, cont.

\begin{tabular}{|c|c|c|c|c|c|c|c|c|c|c|}
\hline Local/Espécie & Família & $\begin{array}{c}\mathrm{N}^{\mathrm{o}} \\
\text { Ind. }\end{array}$ & $\begin{array}{c}\mathrm{N}^{\mathrm{o}} \\
\text { Parc. }\end{array}$ & Fre & Den & Abu & Frr & Der & Abr & IVI \\
\hline \multicolumn{11}{|l|}{ Iguatama } \\
\hline Ageratum conyzoides $\mathrm{L}$. & Asteraceae & 264 & 78 & 0,78 & 10,56 & 3,38 & 20,86 & 31,43 & 2,83 & 55,12 \\
\hline Cyperusrotundus L. & Cyperaceae & 144 & 20 & 0,20 & 5,76 & 7,20 & 5,35 & 17,14 & 6,03 & 28,52 \\
\hline Sida urens $\mathrm{L}$. & Malvaceae & 57 & 34 & 0,34 & 2,28 & 1,68 & 9,09 & 6,79 & 1,40 & 17,28 \\
\hline Digitaria horiizontalis Willd. & Poaceae & 55 & 8 & 0,08 & 2,20 & 6,88 & 2,14 & 6,55 & 5,76 & 14,44 \\
\hline Ipomoea grandifolia O'Don & Convolvulaceae & 32 & 28 & 0,28 & 1,28 & 1,14 & 7,49 & 3,81 & 0,96 & 12,25 \\
\hline Chloris sp. & Poaceae & 10 & 1 & 0,01 & 0,40 & 10,00 & 0,27 & 1,19 & 8,37 & 9,83 \\
\hline Leonotis nepetifolia (L.) W.T. Aiton & Lamiaceae & 27 & 17 & 0,17 & 1,08 & 1,59 & 4,55 & 3,21 & 1,33 & 9,09 \\
\hline Phyllanthus tenellus Roxb. & Euphorbiaceae & 22 & 19 & 0,19 & 0,88 & 1,16 & 5,08 & 2,62 & 0,97 & 8,67 \\
\hline Ipomoea hederifolia $\mathrm{L}$. & Convolvulaceae & 22 & 17 & 0,17 & 0,88 & 1,29 & 4,55 & 2,62 & 1,08 & 8,25 \\
\hline Althernanthera tenella Colla & Amaranthaceae & 17 & 16 & 0,16 & 0,68 & 1,06 & 4,28 & 2,02 & 0,89 & 7,19 \\
\hline \multicolumn{11}{|l|}{ Lagoa da Prata } \\
\hline Blainvillea biaristata DC. & Asteraceae & 102 & 50 & 0,50 & 4,08 & 2,04 & 5,43 & 12,36 & 2,48 & 20,28 \\
\hline Commelina benghalensis L. & Commelinaceae & 67 & 49 & 0,49 & 2,68 & 1,37 & 5,33 & 8,12 & 1,66 & 15,11 \\
\hline Ageratum conyzoides $\mathrm{L}$. & Asteraceae & 66 & 30 & 0,30 & 2,64 & 2,20 & 3,26 & 8,00 & 2,67 & 13,94 \\
\hline Sida carpiniifolia L f. & Malvaceae & 58 & 38 & 0,38 & 2,32 & 1,53 & 4,13 & 7,03 & 1,86 & 13,02 \\
\hline Elvira biflora (L.) DC. & Asteraceae & 51 & 32 & 0,32 & 2,04 & 1,59 & 3,48 & 6,18 & 1,94 & 11,60 \\
\hline Sida glasiovii Schum. & Malvaceae & 47 & 32 & 0,32 & 1,88 & 1,47 & 3,48 & 5,70 & 1,79 & 10,96 \\
\hline Bidens pilosa $\mathrm{L}$. & Asteraceae & 31 & 6 & 0,06 & 1,24 & 5,17 & 0,65 & 3,76 & 6,28 & 10,69 \\
\hline Chamaecrista rotundifolia (Pers) Gree. & Leguminosae-Caes. & 1 & 90 & 0,90 & 0,04 & 0,01 & 9,78 & 0,12 & 0,01 & 9,92 \\
\hline Malvaceae urens $\mathrm{L}$. & Malvaceae & 41 & 29 & 0,29 & 1,64 & 1,41 & 3,15 & 4,97 & 1,72 & 9,84 \\
\hline Chamaessyce hirta (L.) Mill. & Euphorbiaceae & 41 & 22 & 0,22 & 1,64 & 1,86 & 2,39 & 4,97 & 2,27 & 9,63 \\
\hline \multicolumn{11}{|l|}{ Luz } \\
\hline Conyza bonariensis (L.) Cronq. & Asteraceae & 206 & 80 & 0,80 & 8,24 & 2,58 & 15,69 & 25,31 & 3,31 & 44,30 \\
\hline Ageratum conyzoides $\mathrm{L}$. & Asteraceae & 117 & 45 & 0,45 & 4,68 & 2,60 & 8,82 & 14,37 & 3,34 & 26,54 \\
\hline Corchorus hirtus L. & Tiliaceae & 75 & 67 & 0,67 & 3,00 & 1,12 & 13,14 & 9,21 & 1,44 & 23,79 \\
\hline Sida urens $\mathrm{L}$. & Malvaceae & 72 & 59 & 0,59 & 2,88 & 1,22 & 11,57 & 8,85 & 1,57 & 21,98 \\
\hline Leonurus sibiricus L. & Lamiaceae & 72 & 23 & 0,23 & 2,88 & 3,13 & 4,51 & 8,85 & 4,02 & 17,38 \\
\hline Sida viarum St. Hill. & Malvaceae & 49 & 40 & 0,40 & 1,96 & 1,23 & 7,84 & 6,02 & 1,57 & 15,44 \\
\hline Hyptis densiflora Mart. & Lamiaceae & 23 & 16 & 0,16 & 0,92 & 1,44 & 3,14 & 2,83 & 1,85 & 7,81 \\
\hline Sida glasiovii K. Schum. & Malvaceae & 16 & 13 & 0,13 & 0,64 & 1,23 & 2,55 & 1,97 & 1,58 & 6,10 \\
\hline Hyptis suaveolens (L.) Poit. & Lamiaceae & 13 & 10 & 0,10 & 0,52 & 1,30 & 1,96 & 1,60 & 1,67 & 5,23 \\
\hline Hyptis lophantha Mart. ex Bent. & Lamiaceae & 12 & 10 & 0,10 & 0,48 & 1,20 & 1,96 & 1,47 & 1,54 & 4,98 \\
\hline \multicolumn{11}{|l|}{ Dores do Indaiá } \\
\hline Ageratum conyzoides L. & Asteraceae & 363 & 72 & 0,72 & 14,52 & 5,04 & 13,85 & 38,54 & 5,56 & 57,94 \\
\hline Stachytarfeta cayenensis (Rich.) Vahl. & Verbenaceae & 64 & 48 & 0,48 & 2,56 & 1,33 & 9,23 & 6,79 & 1,47 & 17,50 \\
\hline Conyza bonariensis (L.) Cronq. & Asteraceae & 60 & 31 & 0,31 & 2,40 & 1,94 & 5,96 & 6,37 & 2,13 & 14,47 \\
\hline Corchorus hirtus L. & Tiliaceae & 48 & 40 & 0,40 & 1,92 & 1,20 & 7,69 & 5,10 & 1,32 & 14,11 \\
\hline Turnera ulmifolia $\mathrm{L}$. & Turneraceae & 40 & 34 & 0,34 & 1,60 & 1,18 & 6,54 & 4,25 & 1,30 & 12,08 \\
\hline Emilia sonchyfolia DC. & Asteraceae & 39 & 26 & 0,26 & 1,56 & 1,50 & 5,00 & 4,14 & 1,65 & 10,79 \\
\hline Sida urens L. & Malvaceae & 33 & 24 & 0,24 & 1,32 & 1,38 & 4,62 & 3,50 & 1,52 & 9,63 \\
\hline Sida glasiovii K. Schum. & Malvaceae & 23 & 13 & 0,13 & 0,92 & 1,77 & 2,50 & 2,44 & 1,95 & 6,89 \\
\hline Acanthospermum australe (Lf.) Kunt. & Asteraceae & 17 & 10 & 0,10 & 0,68 & 1,70 & 1,92 & 1,80 & 1,87 & 5,60 \\
\hline Heliotropium indicum L. & Boraginacee & 14 & 12 & 0,12 & 0,56 & 1,17 & 2,31 & 1,49 & 1,29 & 5,08 \\
\hline
\end{tabular}


Tabela 2, cont.

\begin{tabular}{|c|c|c|c|c|c|c|c|c|c|c|}
\hline Local/Espécie & Família & $\mathrm{N}^{\mathrm{o}}$ & $\mathrm{N}^{\mathrm{o}}$ & Fre & Den & Abu & Frr & Der & Abr & IVI \\
\hline \multicolumn{11}{|l|}{ Bom Despacho } \\
\hline Gaya sp. & Malvaceae & 143 & 80 & 0,80 & 5,72 & 1,79 & 15,27 & 20,58 & 2,30 & 38,14 \\
\hline Sida urens L. & Malvaceae & 80 & 49 & 0,49 & 3,20 & 1,63 & 9,35 & 11,51 & 2,10 & 22,96 \\
\hline Sida santaremnensis Monteiro & Malvaceae & 90 & 34 & 0,34 & 3,60 & 2,65 & 6,49 & 12,95 & 3,40 & 22,8 \\
\hline Heliotropium indicum $\mathrm{L}$. & Boraginaceae & 2 & 97 & 0,97 & 0,08 & 0,02 & 18,51 & 0,29 & 0,03 & 18,83 \\
\hline Sida glasiovii K. Schum. & Malvacee & 57 & 18 & 0,18 & 2,28 & 3,17 & 3,44 & 8,20 & 4,07 & 15,71 \\
\hline Conyza bonariensis (L.) Cronq. & Asteraceae & 38 & 23 & 0,23 & 1,52 & 1,65 & 4,39 & 5,47 & 2,12 & 11,98 \\
\hline Chamaessyce hirta (L.) Mill. & Euphorbiacee & 34 & 25 & 0,25 & 1,36 & 1,36 & 4,77 & 4,89 & 1,75 & 11,4 \\
\hline Eragrostes sp.1 & Poaceae & 35 & 11 & 0,11 & 1,40 & 3,18 & 2,10 & 5,04 & 4,09 & 11,23 \\
\hline Ageratum conyzoides L. & Asteraceae & 18 & 16 & 0,16 & 0,72 & 1,13 & 3,05 & 2,59 & 1,45 & 7,09 \\
\hline Emilia sonchyfolia DC & Asteraceae & 18 & 16 & 0,16 & 0,72 & 1,13 & 3,05 & 2,59 & 1,45 & 7,05 \\
\hline \multicolumn{11}{|l|}{ Martinho Campos } \\
\hline Ageratum conyzoides $\mathrm{L}$. & Asteraceae & 297 & 89 & 0,89 & 11,88 & 3,34 & 18,39 & 32,57 & 5,21 & 56,17 \\
\hline Brachiaria sp.1 & Poaceae & 136 & 62 & 0,62 & 5,44 & 2,19 & 12,81 & 14,91 & 3,43 & 31,15 \\
\hline Sida sp.1 & Malvaceae & 135 & 59 & 0,59 & 5,40 & 2,29 & 12,19 & 14,80 & 3,57 & 30,57 \\
\hline Sida glasiovii K. Schum. & Malvaceae & 53 & 28 & 0,28 & 2,12 & 1,89 & 5,79 & 5,81 & 2,96 & 14,55 \\
\hline Emilia sonchyfolia DC. & Asteraceae & 37 & 28 & 0,28 & 1,48 & 1,32 & 5,79 & 4,06 & 2,06 & 11,9 \\
\hline Chamaessyce hirta (L.) Mill. & Euphorbiaceae & 30 & 26 & 0,26 & 1,20 & 1,15 & 5,37 & 3,29 & 1,80 & 10,46 \\
\hline Sida urens $\mathrm{L}$. & Malvaceae & 23 & 21 & 0,21 & 0,92 & 1,10 & 4,34 & 2,52 & 1,71 & 8,57 \\
\hline Cyperus rotundus $\mathrm{L}$. & Cyperaceae & 18 & 6 & 0,06 & 0,72 & 3,00 & 1,24 & 1,97 & 4,68 & 7,90 \\
\hline Vernonia polyanthes Lees. & Asteraceae & 16 & 11 & 0,11 & 0,64 & 1,45 & 2,27 & 1,75 & 2,27 & 6,30 \\
\hline Paspalum notatum Fliigge & Poaceae & 14 & 13 & 0,13 & 0,56 & 1,08 & 2,69 & 1,54 & 1,68 & 5,90 \\
\hline \multicolumn{11}{|l|}{ Pompéu } \\
\hline Sida glasiovii K. Schum. & Malvaceae & 371 & 89 & 0,89 & 14,84 & 4,17 & 17,45 & 36,81 & 3,96 & 58,22 \\
\hline Calopogonium mucunoides Desv. & Leguminosae-Pap. & 68 & 13 & 0,13 & 2,72 & 5,23 & 2,55 & 6,75 & 4,97 & 14,26 \\
\hline Echinochloa cruz-gali (L.) Beauv. & Poaceae & 70 & 21 & 0,21 & 2,80 & 3,33 & 4,12 & 6,94 & 3,17 & 14,23 \\
\hline Sida urens L. & Malvacee & 49 & 35 & 0,35 & 1,96 & 1,40 & 6,86 & $\begin{array}{l}4,86 \\
4,8\end{array}$ & 1,33 & 13,05 \\
\hline Chamaesyce hirta (L.) Mill. & Euphorbiacee & 48 & 29 & 0,29 & 1,92 & 1,66 & 5,69 & 4,76 & 1,57 & 12,02 \\
\hline Ageratum conyzoides $\mathrm{L}$. & Asteraceae & 38 & 23 & 0,23 & 1,52 & 1,65 & 4,51 & 3,77 & 1,57 & 9,85 \\
\hline Malvastrum coromandelianum (L.) Ga. & Malvaceae & 35 & 26 & 0,26 & 1,40 & 1,35 & 5,10 & 3,47 & 1,28 & 9,85 \\
\hline Sida rhombifolia L. & Malvaceae & 25 & 17 & 0,17 & 1,00 & 1,47 & 3,33 & 2,48 & 1,40 & 7,21 \\
\hline Digitaria horizontalis Willd. & Poaceae & 20 & 20 & 0,20 & 0,80 & 1,00 & 3,92 & 1,98 & 0,95 & 6,86 \\
\hline Corchorus hirtus L. & Tiliaceae & 20 & 11 & 0,11 & 0,80 & 1,82 & 2,16 & 1,98 & 1,73 & 5,87 \\
\hline \multicolumn{11}{|l|}{ Abaeté } \\
\hline Sida rhombifolia $\mathrm{L}$. & Malvaceae & 103 & 43 & 0,43 & 4,12 & 2,40 & 7,72 & 13,68 & 2,29 & 23,65 \\
\hline Ageratum conyzoides L. & Asteraceae & 75 & 44 & 0,44 & 3,00 & 1,70 & 7,90 & 9,96 & 1,63 & 19,4 \\
\hline Sida glasiovii K. Schum. & Malvaceae & 74 & 30 & 0,30 & 2,96 & 2,47 & 5,39 & 9,83 & 2,36 & 17,57 \\
\hline Ipomoea grandifolia (D.) O,Don & Convolvulaceae & 48 & 44 & 0,44 & 1,92 & 1,09 & 7,90 & 6,37 & 1,04 & 15,32 \\
\hline Brachiaria mutica (Forst.) Stapf. & Poaceae & 47 & 41 & 0,41 & 1,88 & 1,15 & 7,36 & 6,24 & 1,10 & 14,70 \\
\hline Cynidin dactylon & Poaceae & 42 & 42 & 0,42 & 1,68 & 1,00 & 7,54 & 5,58 & 0,96 & 14,07 \\
\hline Senna obtusifolia (L.) Irwin \& Barneby & Leguminosae-Caes. & 46 & 32 & 0,32 & 1,84 & 1,44 & 5,75 & 6,11 & 1,38 & 13,23 \\
\hline Sida urens L. & Malvaceae & 42 & 35 & 0,35 & 1,68 & 1,20 & 6,28 & 5,58 & 1,15 & 13,01 \\
\hline Brachiaria ruziziensis sGer. \& Evr. & Poaceae & 30 & 25 & 0,25 & 1,20 & 1,20 & 4,49 & 3,98 & 1,15 & 9,62 \\
\hline Sida cordifolia $\mathrm{L}$. & Malvaceae & 14 & 11 & 0,11 & 0,56 & 1,27 & 1,97 & 1,86 & 1,22 & 5,05 \\
\hline
\end{tabular}


Embora as espécies mais importantes se concentrem em apenas duas famílias (Asteraceae e Malvaceae), os índices de similaridade calculados entre as áreas evidenciam diferenças florísticas entre elas (Tabela 3).

Tabela 3 - Índices de similaridade calculados entre as áreas estudadas nas margens do Rio São Francisco. 1 = Vargem Bonita; 2 = Piumhi; 3 = Doresópolis; 4 = Bambuí; $5=$ Iguatama; 6 = Lagoa da Prata; $7=$ Luz; $8=$ Dores do Indaiá; $9=$ Bom Despacho; $10=$ Martinho Campos; $11=$ Pompéu; 12 = Abaeté

\begin{tabular}{|r|c|c|c|c|c|c|c|c|c|c|c|}
\hline & 2 & 3 & 4 & 5 & 6 & 7 & 8 & 9 & 10 & 11 & 12 \\
\hline 1 & 0,27 & 0,37 & 0,26 & 0,18 & 0,33 & 0,27 & 0,40 & 0,28 & 0,44 & 0,43 & 0,34 \\
2 & & 0,14 & 0,24 & 0,24 & 0,21 & 0,17 & 0,17 & 0,10 & 0,21 & 0,25 & 0,21 \\
3 & & & 0,48 & 0,35 & 0,41 & 0,44 & 0,42 & 0,45 & 0,38 & 0,35 & 0,44 \\
4 & & & & 0,38 & 0,38 & 0,43 & 0,43 & 0,43 & 0,36 & 0,36 & 0,45 \\
5 & & & & & 0,42 & 0,47 & 0,38 & 0,38 & 0,34 & 0,34 & 0,38 \\
6 & & & & & & 0,45 & 0,41 & 0,38 & 0,37 & 0,32 & 0,41 \\
7 & & & & & & & 0,48 & 0,42 & 0,37 & 0,41 & 0,46 \\
8 & & & & & & & & 0,53 & 0,53 & 0,47 & 0,59 \\
9 & & & & & & & & & 0,52 & 0,41 & 0,48 \\
10 & & & & & & & & & & 0,50 & 0,48 \\
11 & & & & & & & & & & & 0,47 \\
\hline
\end{tabular}

As espécies dominantes em um local nem sempre o são em outro, o que permite utilizar herbicidas específicos para controlar apenas as espécie mais abundantes, mantendo a sustentabilidade no local. Como reflexo das características climáticas e das práticas agronômicas adotadas, manejo do solo e aplicação de herbicidas, em anos subseqüentes, poderá haver alteração na dinâmica populacional, surgindo outras espécies dominantes (Godoy et al., 1995).

Os herbicidas controlam as plantas daninhas por um período, mas não evitam a emergência tardia de muitas delas; no caso do milho, as plantas que emergem tardiamente podem prejudicar a colheita. Isso constou do relato de produtores que usaram herbicidas aplicados em pós-emergência, e, mesmo assim, a ocorrência de corda-de-viola (Ipomoea sp.) foi tão intensa que impediu a colheita mecânica. Outros disseram que, ao fazerem o controle químico, não encontraram herbicidas capazes de controlar com eficácia as plantas conhecidas por trapoeraba (Commelinaceae), devendo-se ressaltar que a espécie Commelina benghalensis é comum na área estudada e teve alto IVI em Lagoa da Prata $(15,11)$. No entanto, é possivel formular misturas de herbicidas que controlam esta espécie (Ronchi et al., 2002).

\section{LITERATURA CITADA}

BULHER, D. D. et al. Integrating mechanical weeding with reduced herbicide use in conservation tillage corn production systems. Agron. J., v. 87, p. 507-512, 1995.

CRUTCHFIELD, D. A.; WICKS, G. A.; BURNISE, O. C. Effect of winter wheat (Triticum aestivum) straw mulch level on weed control. Weed Sci., v. 34, p. 110-114, 1986.

GODOY, G.; VEGA, J.; PITTY, A. El tipo de labranza afecta la flora y la distribución vertical del banco de semillas de malezas. Ceiba, v. 36, n. 2, p. 217-229, 1995.

GUERSA, C. M.; MARTÍNEZ-GUERSA, M. A. Ecological correlates of seed size and persistence in the soil under different tilling systems: implications for weed management. Field Crops Res., v. 67, p. 141-148, 2000.

JOHNSON, M. D.; WYSE, D. L.; LUESCHEN, W. E. The influence of herbicide formulation on weed control in four tillage systems. Weed Sci., v. 37, p. 239-249, 1989.

MACEDO, J. F.; MARTINS, R. P. Potencial da erva daninha Waltheria americana (Sterculiaceae) no manejo integrado de pragas e polinizadores: visitas de abelhas e vespas. An. Soc. Ent. Brasil, v. 27, n. 1, p. 29- 40, 1998.

MONQUERO, P. A.; CHRISTOFOLLETI, P. J.; SANTOS, C. T. D. Glyphosate em mistura com herbicidas alternativos para o manejo de plantas daninhas. Planta Daninha, v. 19, n. 3, p. 375-380, 2001.

MULUGUETA, D.; STOLTEMBERG, D. E. Increase weed emergence and seed bank depletion by soil disturbance in no-tillage systems. Weed Sci., v. 45, p. 234-241, 1997.

ORYOKOT, J. O. E.; MURPHY, S. D.; SWANTON, C. J. Effect of tillage and corn on pigweed (Amaranthus spp.) seedling emergence and density. Weed Sci., v. 45, p. 120126, 1997.

PITELLI, R. A. Estudos fitossociológicos em comunidades infestantes de agroecossistemas. J. Conserb, v. 1, n. 2, p. 1-7, 2000.

RONCHI, C. P. et al. Misturas de herbicidas para o controle de plantas daninhas do gênero Commelina. Planta

Daninha, v. 20, n. 2, p. 311-318, 2002.

VOLL, E. et al. Dinâmica do banco de sementes de plantas daninhas sob diferentes sistemas de manejo do solo. Planta Daninha, v. 19, n. 2, p. 171-178, 2001. 